\title{
Les standards de formation
}

Le cas des États-Unis

Standards for learning: the USA Case

Estándares de formación. El caso de Estados Unidos

\section{S. Paul Reville}

Traducteur : N. Métayer et Marie-José Sanselme

\section{OpenEdition}

\section{Journals}

Édition électronique

URL : http://journals.openedition.org/ries/206

DOI : $10.4000 /$ ries.206

ISSN : 2261-4265

Éditeur

Centre international d'études pédagogiques

Édition imprimée

Date de publication : 1 décembre 2006

Pagination : 23-32

ISSN : $1254-4590$

Référence électronique

S. Paul Reville, "Les standards de formation », Revue internationale d'éducation de Sèvres [En ligne], 43 | décembre 2006, mis en ligne le 22 juin 2011, consulté le 21 avril 2019. URL : http:// journals.openedition.org/ries/206 ; DOI : 10.4000/ries.206 


\section{Les standards de formation}

\section{Le cas des États-Unis*}

\section{S. Paul Reville}

Jusqu'à il y a une vingtaine d'années, les standards ${ }^{1}$ de formation aux États-Unis relevaient uniquement des autorités locales. À cette époque, bon nombre de décideurs du pays traversèrent une grave crise de confiance à l'encontre des écoles publiques. Ceci mena à une remise en cause de la mission de l'instruction publique aboutissant finalement à ce que la quasi intégralité des cinquante États adopte des référentiels de formation relativement clairs, définissant ce que les élèves devaient être capable de réaliser aux différentes étapes de leur éducation. Les États-Unis ont maintenant derrière eux presque deux décennies d'expérience dans le domaine des standards et de l'obligation de responsabilité (standards and accountability). Au cours de cette période, les contenus et les niveaux d'apprentissage sont devenus le centre de multiples et intenses réformes.

\section{FONDEMENTS DU SYSTÈME SCOLAIRE AMÉRICAIN}

Afin de comprendre le mouvement des standards aux États-Unis, il faut d'abord présenter brièvement la nature du pilotage du système éducatif dans un pays où il est hautement décentralisé.

Depuis l'époque coloniale (au début du XVII ${ }^{\mathrm{e}}$ siècle), les citoyens se méfient d'un pouvoir éloigné et centralisé. Ils préférèrent, particulièrement dans le domaine de l'éducation, un système de pilotage qui permet de confier la responsabilité de l'enseignement public aux autorités locales. Alors que la nation évoluait et accédait à son indépendance à la fin du XVIII ${ }^{\mathrm{e}}$ siècle, les «pères fondateurs » rédigèrent et appliquèrent la constitution américaine qui ne faisait aucunement référence à l'enseignement. De ce fait, ils en confiaient délibérément la responsabilité aux différents États. Au XIX siècle, les États commencèrent à assumer davantage de responsabilités en matière d'éducation et à partager les décisions avec les autorités locales ${ }^{2}$. Au milieu du $\mathrm{XX}^{\mathrm{e}}$ siècle, l'État fédéral commença à faire valoir son autorité, mais en assumant seulement

\footnotetext{
* Article traduit par N. Métayer et M.-J. Sanselme.

1. NdIR : la notion de «standards» couvre, aux États-Unis, à peu près le même champ que celle de «programmes d'enseignement » en France. Selon le contexte, le terme sera traduit dans l'article par «programme d'enseignement » ou par «référentiels de formation ».

2. Tyack, David and Cuban, Larry. Tinkering Toward Utopia. (Cambridge, MA : Harvard University Press, 1995).
} 
un rôle secondaire dans un partenariat avec tous les niveaux de l'administration scolaire américaine ${ }^{3}$.

Ce partenariat à divers niveaux constitue le pilotage actuel d'un système scolaire dans lequel cinquante États légifèrent pour définir le cadre dans lequel évoluent 14000 commissions de l'éducation élues localement pour définir la politique et faire fonctionner 93000 établissements représentant approximativement cinquante millions d'élèves ${ }^{4}$. Ce sont maintenant prioritairement les gouvernements des États qui déterminent les standards et les objectifs d'apprentissage pour tous les élèves. Les responsables locaux sont responsables des résultats de leurs établissements et de leurs élèves en fonction des objectifs fixés par chaque État. Ils élaborent également des programmes et complètent les référentiels de formation définis par l'État en y ajoutant leurs propres objectifs. Entre-temps, le gouvernement fédéral, par le biais de la loi No Child Left Behind Act (NCLB), votée en 2001, a encore accru la pression de l'obligation de résultats par rapport aux objectifs définis par chaque État.

\section{LE MOUVEMENT DES STANDARDS}

Un rapport national sur l'éducation intitulé Une Nation en Péril ${ }^{5}$ paraît en 1983. Son contenu, incendiaire, est fort remarqué. Ses auteurs, la commission nationale pour l'excellence scolaire, y utilisent un langage véhément pour remettre en question la complaisance sur la qualité des écoles américaines. "Si une puissance étrangère ennemie avait essayé d'imposer à l'Amérique les performances médiocres en terme d'éducation qu'elle connaît aujourd'hui, on aurait pu considérer cela comme un acte de guerre». "Nous déclarons que si le peuple américain peut s'enorgueillir des réalisations, dans le passé, de ses écoles et de ses universités et de leurs contributions à la nation et au bien-être de ses habitants, une vague grandissante de médiocrité érode les fondements du système scolaire de notre société, menaçant ainsi notre avenir en tant que nation et en tant que peuple ${ }^{6}$.

Ce cri d'alarme capta l'attention de la nation, et en particulier celle des gouverneurs et des chefs d'entreprise qui étaient eux-mêmes préoccupés par les échecs du système scolaire américain quand il était comparé à celui des autres pays. Ils voyaient les écoles comme un moteur vital pour le développement économique dans un monde hautement concurrentiel. Ils constataient que le système éducatif en place était plus adapté aux défis industriels qu'avait connus

3. Kirst, M. and Wirt, F. The Political Dynamics of American Education zed. (Berkeley, CA : McCutchan Publishing, 2005).

4. Kober, Nancy. A Public Education Primer : Basic (and Sometimes Surprising) Facts about the U.S. Education System. (Washington, DC : Center on Education Policy, 2006), www. cep-dc. org.

5. A nation at risk.

6. National Commission on Excellence in Education. A Nation at Risk : The Imperative for Educational Reform. (Washington, DC : Author, 1983). 
le pays au début du $\mathrm{XX}^{\mathrm{e}}$ siècle qu'au défi post-industriel du $\mathrm{XXI}^{\mathrm{e}}$ siècle, à l'ère de la communication. Ils constataient que l'avenir de l'Amérique dépendait du développement de sa compétitivité par rapport aux autres nations industrialisées. Cet avantage serait acquis par le développement d'une force de travail aux compétences et aux connaissances plus élevées que celles des autres pays. Pour de nombreux décideurs, il s'agissait là d'une question de survie. L'un des rapports les plus provocateurs de l'époque s'intitulait Le Choix de l'Amérique : hautes compétences ou bas salaires ${ }^{7}$. Il reflétait l'état d'esprit de décideurs préoccupés par l'automatisation des emplois non qualifiés et par leur exportation vers des pays à la main-d'œuvre bon marché. Les États-Unis n'avaient donc d'autre choix que de "relever le niveau» en augmentant les compétences et les connaissances de tous les travailleurs futurs. Un rapport de la fondation Carnegie s'exprime à cette époque en ces termes : "si nous voulons maintenir notre niveau de vie, si nous voulons éviter l'augmentation d'un quart monde permanent, si nous voulons que notre démocratie fonctionne au cours du siècle à venir, nos écoles doivent diplômer une large majorité d'étudiants dont le niveau ne pouvait, par le passé, être imaginé que pour quelques privilégiés. ${ }^{8}$. C'était un défi important lancé à un système scolaire que bien des dirigeants percevaient comme défectueux.

Les gouverneurs et les chefs d'entreprise s'accordèrent pour dire que le système scolaire présentait un certain nombre de pathologies, quelques unes des plus importantes étant l'absence de comptes à rendre en matière de résultats ; de définition claire du «produit»; l'incohérence d'un système totalement décentralisé; l'absence d'autorité dévolue aux décideurs du système; le manque de systèmes de développement des ressources humaines pour servir une industrie nécessitant une main-d'œuvre nombreuse et hautement qualifiée; et tout particulièrement, l'absence d'objectifs clairement définis, conséquence d'un élargissement insidieux des types de mission censées être remplies par les écoles pour répondre à une foule de besoins universitaires, sociaux, récréatifs, médicaux, nutritionnels ou autres si bien que leurs missions étaient devenues confuses et leurs responsabilités accablantes. De ce point de vue, la première étape de réforme de ce système sclérosé serait de restreindre le champ de ses missions pour resserrer ses tâches, clarifier ses objectifs, utiliser des stratégies visant à augmenter la capacité des employés à travailler à un niveau radicalement plus élevé que celui qu'on attendait d'eux autrefois ${ }^{9}$.

C'est ainsi que naquit le «mouvement des standards». C'était une stratégie de bon sens qui visait les deux pôles historiques de l'éducation américaine,

7. America's Choice: High Skills or Low Wages.

8. Carnegie Forum on Education and the Economy. A Nation Prepared : Teachers for the $21^{\text {st }}$ Century, The Report of the Task Force on Teaching as a Profession. (New York, NY : Author, 1986).

9. Massachusetts Business Alliance for Education. Every Child a Winner : A Proposal for a Legislative Action Plan for Systemic Reform of Massachusetts' Public Primary and Secondary Education System. (Billerica, MA : Author, 1991). 
à savoir l'excellence et l'équité. La logique sous-jacente supposait que les écoles, les éducateurs et finalement les élèves fonctionneraient à des niveaux plus élevés et apprendraient davantage si la mission des écoles publiques était restreinte et clarifiée. Si des référentiels clairs en matière de connaissances et de compétences étaient établis, alors les enseignants, avec l'aide d'un modeste soutien, pourraient concentrer leurs efforts et hisser les élèves au niveau déterminé. L'idée qu'on attendrait de hautes compétences de tous les élèves était la pierre angulaire du mouvement des standards et une orientation radicalement opposée aux pratiques anciennes qui avaient favorisé le développement local de tous les programmes d'enseignement.

Ces référentiels de formation, quand ils existaient, demandaient généralement moins aux élèves de familles modestes ou issus de minorités. Les partisans du système des standards soutenaient que tous les élèves étaient capables d'atteindre, à l'aide de soutiens adaptés, un haut niveau de compétences et de connaissances. Dans cette optique, les éducateurs devaient simplement fournir les conditions nécessaires à un bon apprentissage et s'assurer que chaque enfant ait «l'occasion d'apprendre» selon ces nouveaux programmes d'enseignement. L'accès de tous les élèves à de hauts niveaux devint un impératif moral, éducatif et économique.

Ainsi, la réforme de l'éducation américaine adopta graduellement la devise «un niveau élevé pour tous». Une fois ce niveau fixé, ce que bien des défenseurs de la réforme pensaient à tort être un processus aisé, la théorie faisait appel au développement de programmes adaptés au niveau à atteindre, à une formation continue des professeurs, et à des évaluations régulières et adaptées au niveau attendu, se terminant par un contrôle des résultats allant de l'assistance technique à la sanction.

Les questions suivantes étaient : «quels standards» et «qui sera chargé de les établir?». Des réponses furent beaucoup plus facilement trouvées à cette dernière question qu'à la première. Par le biais d'une combinaison de réformes législatives dans chaque État, d'incitations, de directives fédérales et de quelques décisions d'ordre juridique, il apparut rapidement que la prérogative de l'établissement des standards reviendrait aux gouvernements des États. Les États confièrent pour la plupart aux commissions scolaires la responsabilité d'établir les standards et de mettre en place le processus de développement et d'approbation. De nombreuses commissions tablèrent sur l'hypothèse que des adultes n'auraient que peu ou pas de difficultés à se mettre d'accord sur ce que les enfants doivent savoir et savoir faire. Cependant, en réalité, les méthodes relativement ouvertes que les conseils établirent pour travailler sur la définition des standards générèrent un large éventail d'opinions sur ce qui devrait être inclus dans les objectifs éducatifs de chaque État. Le processus fut plus long, plus empreint d'animosités et source de divisions que cela n'avait été envisagé. Des «guerres de tranchées idéologiques» éclatèrent sur des terrains tels que les 
sciences sociales et l'anglais alors qu'une armée de groupes différents cherchait à faire inclure ses positions dans les standards. Des guerres intestines entre spécialistes de l'éducation en matière de stratégie éducative dévastèrent des domaines comme les mathématiques ou la lecture. Finalement, des cessez-le-feu furent déclarés, on parvint à des compromis et des standards furent établis. Souvent, ces documents étaient victimes de rajouts, ce qui les rendait plus longs et plus exhaustifs que les réformateurs ne l'avaient envisagé auparavant. Ceuxci étaient, à l'origine, à la recherche d'un petit nombre d'objectifs cadrés et mesurables qui mettraient l'accent non pas sur les stratégies pédagogiques mais sur les résultats.

\section{LE CAS DU MASSACHUSETtS}

S’il est impossible de faire des généralisations sur les standards établis dans chacun des cinquante États, l'État du Massachusetts constitue un bon exemple, ses standards étant régulièrement considérés comme parmi les meilleurs du pays. Le processus mis en place dans le Massachusetts est unique, mais bien de ses caractéristiques sont représentatives de celles des autres États ${ }^{10}$.

Le Massachusetts a commencé par établir un Common Core of Learning ${ }^{11}$, document destiné à résumer les connaissances, capacités et qualités de l'élève idéal à la fin de la classe de terminale ${ }^{12}$. Cet ensemble d'apprentissages centraux avait pour but de décrire le type de résultats requis pour préparer un jeune diplômé de n'importe quel lycée du Massachusetts à être admis et à réussir dans des programmes universitaires rigoureux et ce, sans besoin de soutien. Plus largement, l'État souhaitait préparer les jeunes gens à l'éducation tout au long de la vie. Ce Common Core visait également à décrire le bagage nécessaire aux élèves pour qu'ils entrent dans le monde économique soit immédiatement, soit dans l'avenir, et puissent occuper des emplois prometteurs leur assurant un niveau de vie correspondant au moins à celui de la classe moyenne. Les élèves devaient être également préparés à la citoyenneté, à devenir des chefs de file de la démocratie et, s'ils le souhaitaient, des chefs de famille. Le processus d'harmonisation des points de vue sur ce qu'il convenait d'inclure dans le Common Core occasionna des audiences publiques, de multiples ébauches, des témoignages de centaines de personnes de divers horizons et des dizaines de milliers de messages écrits ou électroniques sollicités par la Commission sur l'éducation de l'État ${ }^{13}$.

10. L'auteur de cet article fut membre de la Commission d'éducation du Massachusetts durant les premières années de son travail sur l'établissement des critères.

11. Ndlr : Common Core of learning : «tronc commun de connaissances», ou «ensemble d'apprentissages centraux».

12. Massachusetts Board of Education. Common Core of Learning. (Boston, MA : Author, 1994).

13. NdT : Board and State Department of Education. 
Le Common Core était délibérément conçu comme un document de cadrage général. Les modalités de mise en œuvre seraient détaillées dans une série de documents d'accompagnement intitulés Curriculum Frameworks et qui étaient au nombre de sept, un pour chaque matière : anglais, mathématiques, sciences, technologie, sciences sociales, langues étrangères, hygiène de vie et art. Comme pour le Common Core, on chercha à consulter le plus grand nombre. De nombreux districts régionaux, avec le soutien de l'État, établirent des commissions de professeurs fournissant de longues listes de recommandations à inclure dans le document d'accompagnement ${ }^{14}$. Tant les professeurs que les administrateurs des universités de même que les représentants du monde des affaires y participèrent. Le ministère de l'éducation chercha un échantillonnage de critères exemplaires au niveau national et international. Finalement le ministère proposa et les commissions débattirent, modifièrent et approuvèrent des programmes d'enseignement (Standards) sous forme de documents d'accompagnement (Curriculum Frameworks) détaillés qui servirent de base au développement de documents locaux d'accompagnement, de développement et à l'établissement, au niveau de l'État, d'un système d'évaluation ayant pour but de mesurer les progrès accomplis dans l'acquisition des standards.

Dans le Massachusetts comme dans la plupart des États, il n'existe pas de programmes d'enseignement communs obligatoires. L'État a la responsabilité d'exprimer clairement ses attentes en matière de résultats dans le domaine de l'éducation, de dire ce que les éducateurs et les écoles doivent réaliser avec leurs élèves, pendant que les éducateurs sur le terrain doivent décider de la meilleure démarche à adopter pour que leurs élèves maîtrisent les contenus. Cette approche illustre les bases sous-jacentes de la réforme des standards, c'està-dire une approche à la fois rigide et souple des rapports de responsabilité entre l'État et le niveau local. L'État est clair et vigilant en matière de résultats; il se montre discret en matière de mise en œuvre des programmes et d'instructions données aux autorités locales et aux éducateurs.

\section{ApPrentissages À l’éCOLE ET HORS DE L'ÉCOLE}

Les États-Unis utilisent régulièrement des critères et des évaluations pour tenir les enseignants et les élèves pour responsables de la maîtrise des objectifs à atteindre. Ce schéma ne distingue pas les sources des apprentissages, qu'ils aient été menés à l'école ou ailleurs. Cependant, les référentiels doivent définir un corpus de connaissances et de compétences dans lesquels l'élève doit exceller avant d'aborder le cycle suivant de son éducation. Les éducateurs sont donc chargés de concevoir des systèmes d'enseignement incluant programmes,

14. French, Dan. «The State's Role in Shaping a Progressive Vision of Public Education.» Phi Delta Kappan vol. 80 (1998). 
valeur de l'enseignement, régularité des évaluations, et évaluation du temps nécessaire à l'élève pour qu'il atteigne le niveau requis. $\mathrm{Si}$, par hasard, quelques élèves entrent à l'école avec de l'avance par rapport aux objectifs définis à leur niveau, ils sont cependant en droit de recevoir une formation à la hauteur de leurs attentes. Si d'autres élèves abordent l'école avec peu d'acquis préalables, il incombe au système scolaire de les soutenir, à l'aide des ressources adaptées fournies par l'État.

Certains décideurs éducatifs et experts en évaluation œuvrent activement pour concevoir des outils d'évaluation qui mesureraient la "valeur ajoutée » par l'expérience scolaire de chaque enfant. C'est une entreprise légitime si l'on cherche à établir un véritable système d'évaluation ${ }^{15}$. Néanmoins, les difficultés de la mise en place d'un tel système sont considérables. Même les meilleurs systèmes de mesure de la "valeur ajoutée» ou de la «croissance» ne sont capables de mesurer l'apprentissage des élèves qu'au cours d'une période donnée, et sans entrer dans le détail, sans pouvoir identifier les origines de cet apprentissage.

Pour finir, l'objectif assigné à chaque élève est la maitrise des savoirs. Certains élèves bénéficient pour cela d'un avantage considérable en raison de tout l'éventail d'opportunités externes, formelles ou informelles, que des parents aisés peuvent fournir à leurs enfants. Idéalement, il devrait relever de la responsabilité des écoles de compenser l'absence de ces atouts chez les jeunes défavorisés en leur fournissant une éducation formelle plus intensive et globale. Cela suppose une différenciation, en termes de qualité d'instruction et d'expérience éducative, selon le niveau de maîtrise de chaque élève. Dans un système idéal, chacun devrait pouvoir accéder à un niveau minimum élevé, et le temps réservé à l'enseignement devrait être modulé selon les besoins de chacun. Les États-Unis rencontrent bien des obstacles pour mettre en place un système de ce type mais telle est l'ambition.

\section{ÉVALUATION ET RESPONSABILITÉ : LES DÉFIS}

Une fois le processus d'identification des standards achevé, les réformateurs doivent se tourner vers la mesure des progrès par rapport aux standards. Ces mesures sont effectuées à plusieurs desseins. Le premier consiste à évaluer si les établissements ont effectivement mis en place des programmes centrés sur l'acquisition des standards. Il a aussi un but de diagnostic important afin d'évaluer l'étendue et la profondeur de l'apprentissage, et de permettre aux enseignants d'identifier les besoins les plus importants de leurs élèves en matière d'apprentissage. Ce diagnostic est essentiel pour qu'en fin de compte

15. Crane, Jonathan. The promise of value added testing. (Washington, DC : Progressive Policy Institute, 200o), http: //www.ppionline.org/documents/Value_Added_Testing.pdf. 
tous les élèves accèdent au niveau requis. Les enseignants doivent connaître les statistiques et examiner les travaux des élèves. Ce travail les renseigne sur les forces et les faiblesses du processus d'apprentissage des élèves. Les enseignants peuvent utiliser ces données pour mettre en place des interventions pédagogiques, changer d'approche et répondre ainsi aux besoins diagnostiqués chez les élèves. Dans un système basé sur les standards, ce type de pilotage par les enseignants, renouvelé en permanence de façon professionnelle, centré sur les résultats des élèves et sur l'efficacité des stratégies pédagogiques, devrait devenir la norme.

Un système et des outils d'évaluation de qualité fourniront des informations précises et datées qui pourront être utilisées par les enseignants pour changer et améliorer leurs pratiques pédagogiques. Ces évaluations devraient être fiables et d'utilisation aisée. Dans l'idéal, les outils d'évaluation doivent être aussi riches et divers dans leur approche que les objectifs de résultats qu'ils cherchent à mesurer. Les résultats d'une telle évaluation devraient être compréhensibles et "opératoires", c'est-à-dire qu'ils devraient servir de guide pour améliorer ultérieurement l'enseignement.

Le but final de l'évaluation est de fournir des données susceptibles de renseigner un système qui tient différents intervenants - les équipes administratives, les enseignants et les élèves - pour responsables de la qualité de leur contribution au processus de formation. Ce système de responsabilisation et de responsabilité informe les différentes parties prenantes du processus éducatif - y compris les décideurs, les parents, les équipes administratives, les autorités en place, les enseignants et les élèves - non seulement du niveau des acquis des élèves à un instant donné, mais également de leurs taux de progrès à intervalles réguliers.

Dans le domaine de l'évaluation et des responsabilités, les États-Unis font face à un certain nombre de défis. Le premier est la question de la fiabilité et de la validité de nombreux outils de mesure à grande échelle. Il existe relativement peu d'outils d'évaluation formative, c'est-à-dire d'outils susceptibles de renseigner les enseignants, de préférence régulièrement, pendant l'année scolaire. Nous n'avons pas fait assez de progrès dans le développement de systèmes d'évaluation capables de mesurer la croissance des acquisitions des élèves dans le temps. Certains critères, faibles, entraînent inévitablement des évaluations faibles. Dans d'autres cas, les évaluations elles-mêmes sont défectueuses, simplistes ou en mauvaise adéquation avec les standards. Parfois les enjeux liés aux résultats des élèves sont inadéquats. Ces difficultés ainsi que d'autres, d'ordre technique, sont brûlantes mais surmontables.

Le plus grand défi qui se présente aux éducateurs en matière d'évaluation est la question de savoir ce qu'il convient de faire des résultats disponibles. Comment changer les pratiques pédagogiques et comment forger la capacité à exploiter ces données non seulement pour changer les pratiques mais pour 
concevoir des actions de remédiation permettant de remettre à niveau des écoles ou des systèmes scolaires entièrement déficients? Depuis la promulgation de la nouvelle loi fédérale (NCLB), des dizaines d'établissements sont classés négativement par ces systèmes d'évaluation. Les autorités fédérales ainsi que les États et les autorités locales sont alors désemparés quant à la manière de remédier aux difficultés identifiées par les systèmes de responsabilité ${ }^{16}$.

Des informations de qualité sur le niveau des élèves sont le premier ingrédient dans un système conçu pour aider les enseignants et les systèmes scolaires à pourvoir aux besoins d'apprentissage de tous les élèves. La construction des capacités nécessite dès lors du temps, de la formation continue et une action concertée entre les enseignants pour mettre au point les stratégies individuelles et collectives nécessaires et faire progresser tous les élèves vers une compétence optimale.

Les systèmes d'évaluation nationaux et internationaux peuvent fournir de précieuses informations aux décideurs et aux praticiens. Cependant, la plupart de ces évaluations ne sont que partiellement en adéquation avec les critères qui guident les systèmes éducatifs de chaque État et de chaque commune. Ce manque d'adéquation peut avoir pour conséquence des comparaisons alarmantes, simplement parce que les élèves d'un pays ont passé plus de temps sur une matière ou ont couvert plus tôt un sujet que ceux d'un autre pays. Ces signaux mal interprétés peuvent engendrer une euphorie ou une consternation injustifiée. C'est la raison pour laquelle ils doivent être analysés avec précaution. Cependant, exploitées comme il convient, ces évaluations peuvent fournir de précieuses informations sur les raisons pour lesquelles les élèves d'une zone géographique donnée ont un parcours d'apprentissage différent ou plus réussi que ceux d'une autre région. Elles peuvent être précieuses pour guider à la fois les politiques éducatives et les pratiques pédagogiques.

Les Américains sont des personnes pratiques. Pour eux, l'éducation est un bien pour l'individu comme pour la société. Ils considèrent qu'elle est une préparation essentielle pour une vie réussie. Ils sont conscients de la valeur économique grandissante de l'éducation dans une économie requérant connaissances et compétences de haut niveau. Pour eux, l'éducation est un prérequis pour une économie prospère et ils veulent que leurs enfants soient préparés à prospérer. Pour s'en assurer, les Américains veulent que leurs enfants reçoivent une éducation large à la citoyenneté, au désir d'apprendre tout au long de leur vie, à la compréhension artistique et culturelle, pour ne citer que quelques 
domaines. Mais nombreux sont ceux qui considèrent que le développement de la personnalité et des valeurs relève non de l'école mais de la responsabilité des familles, des communautés religieuses et des cultures locales.

En raison de cette inquiétude sur l'avenir, les Américains ont demandé à leurs établissements scolaires de clarifier ce qu'ils essaient de faire, d'apporter régulièrement la preuve des progrès réalisés, de changer leurs pratiques pédagogiques sur la base des résultats et de rendre compte de leur contribution aux apprentissages. Le mouvement des standards a cherché à expliciter les objectifs fondamentaux de l'éducation en établissant des objectifs ambitieux dans des domaines d'apprentissage essentiels. Ils ont ensuite insisté pour que ces critères ne s'appliquent pas seulement à l'élite mais à tous les élèves. La réforme des standards va droit au cœur du problème : la qualité de l'enseignement et de l'apprentissage dans la classe. Au cours des quinze dernières années, la réforme des standards aux États-Unis a conduit à d'importantes améliorations dans le système mais a simultanément généré beaucoup de résistances et de controverses. Nous avons encore un long chemin à parcourir avant de réaliser notre idéal de réforme scolaire. Maintenant, la priorité du système éducatif américain est de donner les moyens aux établissements de permettre à tous les élèves d'atteindre le niveau requis par les standards. Dans le cas contraire, à long terme, la réforme des standards n'aura été qu'une autre promesse sans lendemain. 